\title{
Investigations of thermal conductivity of simple van der Waals crystal-based nanocomposites
}

\author{
R.V. Nikonkov ${ }^{1}$, P. Stachowiak ${ }^{1}$, T.V. Romanova ${ }^{1}$, A. Jeżowski ${ }^{1}$, and V.V. Sumarokov ${ }^{2}$ \\ ${ }^{1}$ W. Trzebiatowski Institute of Low Temperature and Structure Research, Polish Academy of Sciences \\ 2 Okólna Str., Wroclaw 50-422, Poland \\ ${ }^{2}$ B. Verkin Institute for Low Temperature Physics and Engineering of the National Academy of Sciences of Ukraine \\ 47 Lenin Ave., Kharkov 61103, Ukraine \\ E-mail: P.Stachowiak@int.pan.wroc.pl
}

Received February 3, 2015, published online April 23, 2015

\begin{abstract}
The experimental setup for obtaining and determination of the thermal conductivity of simple van der Waals crystal-based nanocomposites is described. Preliminary thermal conductivity results of measurements carried out in the temperature range 1-40 K on two samples of methane crystals containing nanoparticles of hydroxyapatite are presented. These results confirm usability of the setup and its suitability as a proper experimental method for investigations of the thermal conductivity of the nanocomposites.
\end{abstract}

PACS: $62.23 . \mathrm{Pq}$ Composites (nanosystems embedded in a larger structure);

44.10.+i Heat conduction;

63.20-e Phonons in crystal lattices.

Keywords: thermal conductivity, nanocomposites, cryocrystals, solid methane, hydroxyapatite.

\section{Introduction}

Physical properties of objects with nanometric linear dimensions have been intensely investigated for over ten years. The reason of this interest is twofold:

- basic science: the objects of dimensions of tens of nanometers display new physical properties, usually very much different from their macroscopic analogs;

- possible applications: the new properties of such objects make possible design of new devices. The nanostructured materials along with their new properties applied for new technologies have recently caused an unprecedented acceleration in some fields.

For the similar reason, new complex nanostructured materials are also of great interest and therefore are subject to intensive investigations [1].

A particular example of nanostructured materials are cryocrystals with nanopowders immersed in their volume. A relative simplicity of the crystallographic structure of cryocrystals as matrices and the interactions between the constituents make them ideal objects for basic science investigations. By applying the thermal conductivity experimental technique to investigate such objects, one can get an answer to numerous questions regarding the influence of the properties and parameters of the components of such nanocomposites on their total thermal conductivity. The results of investigations are to determine, in particular, the influence of the size of nanoparticles and their intrinsic transport properties on the resultant thermal conductivity of the nanostructured material. The role of the parameters of the crystalline matrix, such as the interaction strength between its atoms (molecules), the atomic (molecular) mass of the constituents or the type of excited thermal vibrations of the lattice for the thermal conductivity can be specified. In the current contribution we present an experimental technique designed to investigate thermal conductivity of nanopowders of silica, copper and palladium of an average diameter ranging from 10 to $50 \mathrm{~nm}$, embedded in crystalline matrixes of neon, argon, nitrogen and methane. Here we present also some very first results of the introductory measurements carried out on samples of methane containing nanoparticles of hydroxyapatite.

The crystal of methane has been studied for a long time and its thermal conductivity is well known and described. At the equilibrium vapor pressure the crystal of $\mathrm{CH}_{4}$ appears, depending on the temperature, in one of two crystallographic phases. The high-temperature phase I (20.4-90.7 K) features long-range translational ordering and lack of any long-range orientational ordering - the octahedral molecules rotate freely at the fcc lattice sites. With lowering temperature, at $20.4 \mathrm{~K}$ the crystal undergoes a structural phase transition. Below $20.4 \mathrm{~K}$ the crystal exists in phase II. 
In this phase the molecules of methane belong to two subsystems: $75 \%$ of them form six orientationally ordered sublattices while the remaining $25 \%$ belong to another two sublattices. The latter, due to compensating molecular field of the ordered molecules, keep rotating almost freely down to the lowest temperatures.

The hydroxyapatite (HAp), the compound of formula $\mathrm{Ca}_{10}\left(\mathrm{PO}_{4}\right)_{6}(\mathrm{OH})_{2}$, used in the experiment described here was in the form of nanopowder of an average grain size $\sim 30 \mathrm{~nm}$. The substance was obtained by aqueous synthesis and as the structural investigations show it has a crystal symmetry belonging to space group $P 6_{3} / m$.

\section{Experimental}

For the thermal conductivity experiment the LHe cryostat with pumped liquid helium was used. The cryostat enables us to obtain the sample of nanopowders embedded in the structure of a simple cryocrystal and to determine its thermal conductivity with steady-state heat flow method in the temperature range $1-40 \mathrm{~K}$. Both the preparation of the sample and measurements took place directly in the cryostat chamber, without any mechanical manipulations with the sample.

A diagram showing major components of the measuring chamber as well as its photo are shown in Fig. 1(a) and Fig. 1(b), respectively. The central part of the chamber is a glass ampoule of an inner diameter of $6 \mathrm{~mm}$, a wall thickness of $1 \mathrm{~mm}$ and a length of $60 \mathrm{~mm}$. Both ends of the ampoule are closed with copper caps and vacuum tightened with low-temperature epoxy. Before attaching the upper cap, the ampoule was filled with a powder of nanoparticles. The upper cap contains a stainless steel capillary which is used to admit a matrix gas from outside to the cryostat. The cup carries electric heater, along with a thermocouple attached thereto, enables control of the temperature of the upper part of the ampoule during preparation of the matrix crystal. The heater is also used for measurements, for realization of the steady-state heat flow method. For the same purpose, two germanium resistance thermometers are attached to the wall of the ampoule. Before the experiment begins, the ampoule prepared in the way described above is placed inside the measuring chamber of the cryostat: its bottom is connected, both mechanically and thermally, to a copper block of controlled temperature, which can be varied and precisely stabilized in the range 1 to $300 \mathrm{~K}$. After the ampoule with the nanopowder is placed inside the cryostat, it is first thoroughly pumped out. Then the temperature of the ampoule is lowered slightly above the triple point of the matrix substance and the gas is let to the ampoule where the condensation to its liquid phase begins. During the condensation the temperature of the upper part of the ampoule is maintained a few kelvins above the temperature of the bottom so that the liquid gradually fills the ampoule from bottom to top. In this way we obtained a liquefied matrix gas with nanopowder immersed in it. Finally, the temperature of the bottom of the ampoule is lowered, whereupon the liquid solidifies to form a crystal with nanoparticles embedded in its volume.
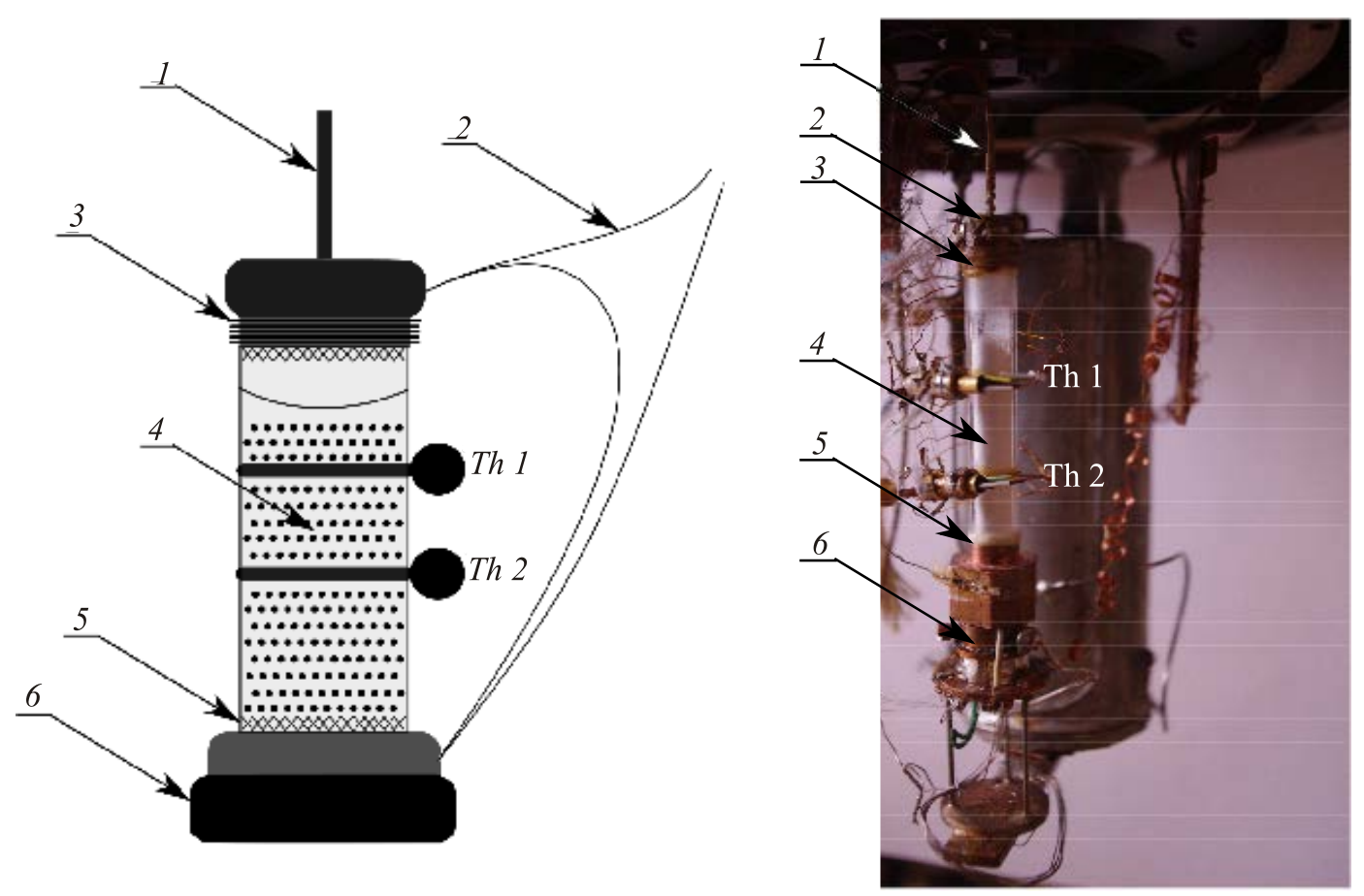

Fig. 1. (Color online) Measuring chamber of the cryostat for obtaining thermal conductivities of simple van der Waals crystal based nanocomposites: diagram (a); photograph (b). 1 is gas feeding capillary, 2 is thermocouple, 3 is gradient heater, 4 is glass ampoule filled with nanopowder, 5 is epoxy, 6 is copper block of controlled temperature, Th1, Th2 are germanium resistance thermometers. 
After crystallization the sample is cooled down to the starting temperature of thermal conductivity measurements. Before measurements, at the temperature $\sim 40 \mathrm{~K}$, helium gas at a pressure of approximately $1 \mathrm{kPa}$ is admitted to the ampoule. The helium serves as a heat exchanging gas which improves the thermal contact of the sample with the gradient heater and the thermometers. As it was verified in a separate experiment, the pressure of the helium does not influence the thermal conductivity results within an appreciable range of its pressure.

In the process of getting the thermal conductivity data, two distorting factors were taken into account: i) The parasitic temperature gradient resulting from the heat radiation due to the temperature mismatch of the LHe thermal shield of the measuring chamber and the sample; and ii) The part of heat transported by the ampoule wall. The former was done by monitoring the temperature values registered by thermometers Th1 and Th2 without heat generated in the gradient heater, while the latter by calibration of the ampoule, i.e., by determining the dependence of thermal conductivity of the empty ampoule, which was carried out in a separate experiment.

\section{Results and discussion}

The results of our measurements are depicted in Fig. 2. The figure displays the temperature dependence of the thermal conductivity of two samples of crystalline methane containing hydroxyapatite nanoparticles of an average grain size of $\sim 30 \mathrm{~nm}$. Here the results for the thermal conductivity of the nanopowder alone are also shown. Similarly to the case of methane-nanoparticle composites, in the

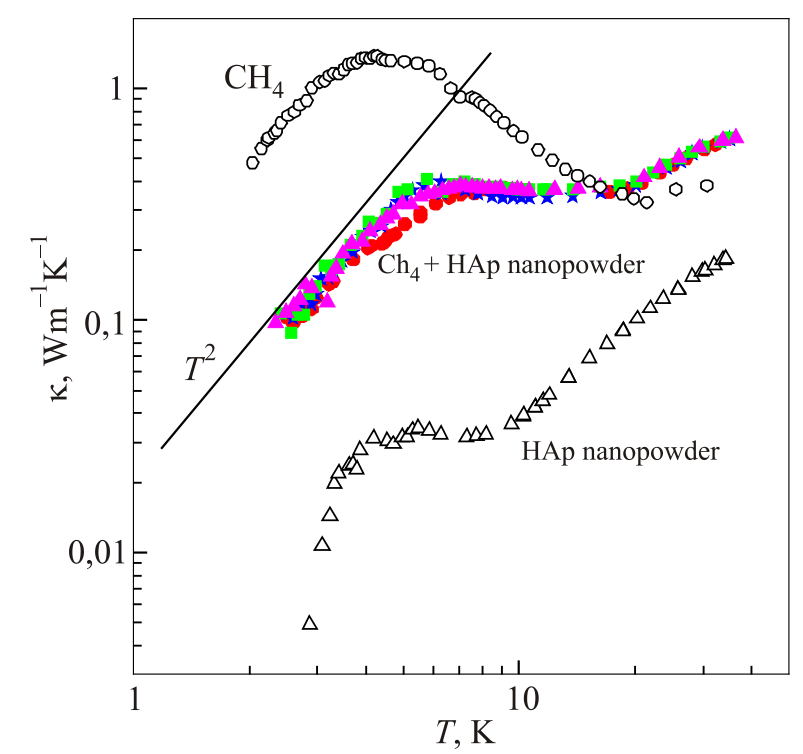

Fig. 2. (Color online) Temperature dependence of the thermal conductivity of two samples of methane-hydroxyapatite nanoparticles nanocomposite (closed symbols). (Open circles) thermal conductivity of methane crystal [2]. (Open triangles) thermal conductivity of hydroxyapatite nanopowder. latter experiment the thermal conductivity of the nanopowder was measured with the heat exchange helium gas present in the ampoule. The earlier results [2] of thermal conductivity of pure crystalline methane are plotted as well.

The thermal conductivity of both nanocomposite samples was determined in two separate temperature walks. Each of the samples, after their preparation, was first cooled down to the lowest achievable temperature $\sim 1 \mathrm{~K}$, whereupon the measurements began. The corresponding data points were obtained with increasing step by step the temperature of the ampoule base. After reaching the temperature of approximately $40 \mathrm{~K}$ the second run started. This time the sample temperature was decreasing. Both walks for both of these two samples gave comparable results. A good agreement of our experimental results obtained for two separate methane-nanopowder of hydroxyapatite samples testifies for right choice of the method of obtaining the composites and therefore applicability of the experimental method.

The temperature dependence of the thermal conductivity of methane-hydroxyapatite nanocomposite resembles to some extent that of disordered solids (see, e.g., [3]). At the lowest temperatures the thermal conductivity varies similarly to glasses, following the dependence $\sim T^{2}$. In the intermediate temperature region, where a typical glass shows a plateau in the thermal conductivity, the thermal conductivity of our nanocomposite decreases with increasing temperature, though the decrease is very weak and the overall dependence resembles a plateau. At a temperature of about $20 \mathrm{~K}$ the thermal conductivity of our samples starts to change similarly to a typical glass, viz., it increases monotonically with increasing temperature.

For the purpose of a rough quantitative analysis of our experimental results we can verify if the thermal conductivity of the investigated heterogeneous structure is determined strictly by the thermal conductivities of its two components and their volume. According to the geometric model commonly used to describe heterogeneous states, the resultant thermal conductivity $\kappa$ of a two-component continuum is the geometric mean of the thermal conductivities of the two components:

$$
\kappa(T)=\kappa_{\text {one }}^{c}(T) * \kappa_{\text {two }}^{d}(T)
$$

where $\kappa, \kappa_{\text {one }}, \kappa_{\text {two }}$ are thermal conductivities of the mixed solid, component one and component two, respectively, $c$ and $d$ are the volume fractions of the component one and component two. In case of the entire volume of the investigated solid completely filled with either of the components, $d=1-c$ [4].

Setting the thermal conductivity of pure methane crystal for $\kappa_{\text {one }}$ and the thermal conductivity of hydroxyapatite nanopowder for $\kappa_{\text {two }}$ we varied $c$ and $d$ parameters to find the best fit of Eq. (1) to the experimental data obtained for the nanocomposite. We found the best match for $c=0.335$ and $d=0.258$ and its plot is depicted in Fig. 3 as the solid 




Fig. 3. (Color online) Thermal conductivity of methane-hydroxyapatite nanoparticles. The solid line is a plot of the best fit of Eq. (1) to the experimental data.

line. As one can see, the best fit cannot be considered satisfactory. Besides, the values of the best fit parameters are far from those one could have expected: The density of hydroxyapatite nanopowder used in our experiments was $0.421 \mathrm{~g} \cdot \mathrm{cm}^{-3}$ while the density of the bulk substance was $3.14 \mathrm{~g} \cdot \mathrm{cm}^{-3}$. From this, one finds that the volume fraction of $\mathrm{Ca}_{10}\left(\mathrm{PO}_{4}\right)_{6}(\mathrm{OH})_{2}$ in our ampoule amounted to 0.134 and after filling it with solid methane the volume fraction of $\mathrm{CH}_{4}$ crystal was 0.866 . Therefore, in conclusion one can state that the thermal conductivity of the nanocomposite is not a simple superposition of thermal conductivities of the methane crystal and the nanopowder and that more complicated phonon mechanisms play a significant role in the thermal transport in such solids.

\section{Acknowledgement}

This work was supported by the National Science Centre (Poland) grant nr. UMO-2013/08/M/ST3/00934.

1. A. Chernatynskiy, D.R. Clarke, and S.R. Phillpot, Handbook of Nanoscience, Engineering, and Technology, Third Edition, Boca Raton, Florida, CRC Press (2012).

2. P. Stachowiak, E. Pisarska, A. Jeżowski, and A.I. Krivchikov, Europhys. Lett. 74, 96 (2006).

3. R.B. Stephens, Phys. Rev. B 13, 852 (1976).

4. A.I. Krivchikov, P. Stachowiak, E. Pisarska, and A. Jeżowski, Phys. Rev. B 75, 012303 (2007). 